\title{
Complementary searches of low mass non-Abelian vector dark matter, dark photon, and dark $Z^{\prime}$
}

\author{
Raymundo Ramos $\oplus^{1, *}$ Tzu-Chiang Yuan $\oplus^{1, \dagger}$ and Van Que Tran $\oplus^{2, *}$ \\ ${ }^{1}$ Institute of Physics, Academia Sinica, Nangang, Taipei 11529, Taiwan \\ ${ }^{2}$ School of Physics, Nanjing University, Nanjing 210093, China
}

(Received 24 January 2021; accepted 30 March 2021; published 19 April 2021)

\begin{abstract}
We investigate a non-Abelian vector dark matter candidate $W^{\prime}$ with a $\mathrm{MeV}-\mathrm{GeV}$ low mass range, accompanied by a dark photon $A^{\prime}$ and a dark $Z^{\prime}$ in the context of a gauged two-Higgs-doublet model. The model is scrutinized by considering various experimental constraints including dark photon searches, electroweak precision data, the relic density of dark matter together with its direct and indirect searches, monojet and Higgs data from the LHC. The viable parameter space indicates that while a dark $Z^{\prime}$ can be the dominant contribution in the relic density due to resonant annihilation of the dark matter, a dark photon is crucial to dark matter direct detection. The model can be further probed in the near future by sub-GeV dark matter and dark photon experiments.
\end{abstract}

DOI: 10.1103/PhysRevD.103.075021

\section{INTRODUCTION}

Albeit mountains of experimental data, ranged from the cosmic microwave background radiation at the largest scale to galaxy structure formation at the local scale, are now providing strong evidences for the existence of dark matter (DM), its particle nature remains elusive. The stability of massive DM is usually implemented in many particle physics models beyond the standard model (SM) by imposing a discrete $Z_{2}$ symmetry in the Lagrangian. The most studied DM candidate is the spin $1 / 2$ lightest neutralino in minimal supersymmetric standard model with $R$ parity [1-3].

Recently, a gauged two-Higgs-doublet model (G2HDM) based on an extended electroweak gauge group $\mathcal{G}=$ $S U(2)_{L} \times U(1)_{Y} \times S U(2)_{H} \times U(1)_{X}$ was proposed [4], in which a hidden discrete $Z_{2}$ symmetry ( $h$ parity) [5] arises naturally as an accidental remnant symmetry rather than imposed ad hoc by hand. This discrete symmetry ensures the stability of the DM candidate in G2HDM, which can be either a complex scalar (in general a linear

\footnotetext{
*raramos@gate.sinica.edu.tw

tcyuan@phys.sinica.edu.tw

*vqtran@nju.edu.cn
}

Published by the American Physical Society under the terms of the Creative Commons Attribution 4.0 International license. Further distribution of this work must maintain attribution to the author(s) and the published article's title, journal citation, and DOI. Funded by SCOAP . combination of various fields in the model as studied in [5]) or a heavy neutrino $\nu^{H}$ or an extra gauge boson $W^{\prime(p, m)}\left(W^{\prime m}=\left(W^{\prime p}\right)^{*}\right)$, all of which have odd $h$ parity. Unlike the left-right symmetric model [6], the $W^{\prime(p, m)}$ in G2HDM do not carry an electric charge. Moreover the emergent $h$ parity ensures there is no tree level flavor changing neutral currents for the SM sector in G2HDM [4], elegantly implementing the notion of natural flavor conservation for neutral currents [7].

The novel idea of G2HDM, as compared with many variants of general 2HDM [8], is that the two Higgs doublets $H_{1}$ and $H_{2}$ of $S U(2)_{L}$ are grouped into a fundamental representation $H=\left(H_{1}, H_{2}\right)^{\mathrm{T}}$ of a new hidden $S U(2)_{H}$. Consistency checks of the model were performed in $[9,10]$. In this work, we will show that the non-Abelian gauge boson $W^{\prime(p, m)}$ associated with $S U(2)_{H}$ can be a viable DM as well. In particular we will focus on the low mass DM scenario in the $\mathrm{MeV}-\mathrm{GeV}$ range and its correlations with two other neutral gauge bosons in $S U(2)_{H} \times U(1)_{X}$.

\section{G2HDM AND ITS MASS SPECTRA}

We begin by simplifying the original G2HDM [4] to remove the $S U(2)_{H}$ triplet scalar $\Delta_{H}$ because it is not absolutely required for a realistic particle spectra and the number of free parameters in the scalar potential can be reduced significantly. New heavy fermions $f^{\mathrm{H}}$ are the same as before due to anomaly cancellations. Details of the model can be found in $[4,9,10]$.

The most general renormalizable Higgs potential invariant under the extended gauge group $\mathcal{G}$ is 


$$
\begin{aligned}
V= & -\mu_{H}^{2}\left(H^{\alpha i} H_{\alpha i}\right)+\lambda_{H}\left(H^{\alpha i} H_{\alpha i}\right)^{2} \\
& +\frac{1}{2} \lambda_{H}^{\prime} \epsilon_{\alpha \beta} \epsilon^{\gamma \delta}\left(H^{\alpha i} H_{\gamma i}\right)\left(H^{\beta j} H_{\delta j}\right) \\
& -\mu_{\Phi}^{2} \Phi_{H}^{\dagger} \Phi_{H}+\lambda_{\Phi}\left(\Phi_{H}^{\dagger} \Phi_{H}\right)^{2}+\lambda_{H \Phi}\left(H^{\dagger} H\right)\left(\Phi_{H}^{\dagger} \Phi_{H}\right) \\
& +\lambda_{H \Phi}^{\prime}\left(H^{\dagger} \Phi_{H}\right)\left(\Phi_{H}^{\dagger} H\right),
\end{aligned}
$$

where $(\alpha, \beta, \gamma, \delta)$ and $(i, j)$ refer to the $S U(2)_{H}$ and $S U(2)_{L}$ indices, respectively, all of which run from one to two, and $H^{\alpha i}=H_{\alpha i}^{*} . \Phi_{H}$ is a $S U(2)_{H}$ doublet in the hidden sector.

To facilitate spontaneous symmetry breaking (SSB), we shift the fields based on our conventional wisdom,

$$
\begin{aligned}
H_{1} & =\left(\begin{array}{c}
H_{11} \\
H_{12}
\end{array}\right)=\left(\begin{array}{c}
G^{+} \\
\frac{v+h}{\sqrt{2}}+i \frac{G^{0}}{\sqrt{2}}
\end{array}\right), \\
\Phi_{H} & =\left(\begin{array}{c}
G_{H}^{p} \\
\frac{v_{\Phi}+\phi_{2}}{\sqrt{2}}+i \frac{G_{H}^{0}}{\sqrt{2}}
\end{array}\right),
\end{aligned}
$$

where $v$ and $v_{\Phi}$ are the vacuum expectation values (VEV)s of $H_{1}$ and $\Phi_{H}$ fields, respectively. $H_{2}=\left(H_{21}, H_{22}\right)^{\mathrm{T}}=$ $\left(H^{+}, H_{2}^{0}\right)^{\mathrm{T}}$ is the inert doublet in G2HDM and does not have VEV. We will work in the 't Hooft-Landau gauge.

The two $h$ parity even fields $h$ and $\phi_{2}$ mix by an angle $\theta_{1}$ satisfying $\tan 2 \theta_{1}=\lambda_{H \Phi} v v_{\Phi} /\left(\lambda_{\Phi} v_{\Phi}^{2}-\lambda_{H} v^{2}\right)$ and give rise to two physical states $h_{1}$ and $h_{2}$. The $h_{1}$ field is identified as the $125 \mathrm{GeV}$ SM-like Higgs boson and $h_{2}$ as a heavier $C P$-even scalar boson. Similarly, the two $h$-parity odd complex fields $G_{H}^{p}$ and $H_{2}^{0 *}$ mix and give rise to two physical states, $\tilde{G}_{H}^{p}$ and $D$. $\tilde{G}_{H}^{p}$ is the massless NambuGoldstone boson absorbed by $W^{\prime p}$, while $D$ is a massive dark complex scalar. The Goldstone bosons $G^{0}, G^{ \pm}$, and $G_{H}^{0}$ are massless. We note that $h_{1,2}, G^{0}, G^{ \pm}$, and $G_{H}^{0}$ are even under $h$ parity, while $\tilde{G}_{H}^{p}, D$ and $H^{ \pm}$are odd [5]. The fact that $H^{ \pm}$has odd $h$ parity implies that the $H^{ \pm} W^{\mp} \gamma$ and $H^{ \pm} W^{\mp} Z$ couplings are absent in G2HDM.

The $W^{ \pm}$gauge boson of $S U(2)_{L}$ remains the same as in $\mathrm{SM}$ with its mass given by $m_{W}=g v / 2$. The $S U(2)_{H}$ gauge boson $W^{\prime(p, m)}$ receives mass from $\left\langle H_{1}\right\rangle$ and $\left\langle\Phi_{2}\right\rangle$,

$$
m_{W^{\prime}}^{2}=\frac{1}{4} g_{H}^{2}\left(v^{2}+v_{\Phi}^{2}\right)
$$

As aforementioned, $W^{\prime(p, m)}$ is odd under $h$ parity, while $W^{ \pm}$is even. If $W^{\prime(p, m)}$ is the lightest $h$-parity odd particle in the model, it can be a stable DM candidate. For earlier discussions of $W^{\prime}$ as DM candidate, see, for example, [11-16].

On the other hand, the SM neutral gauge bosons $B$ and $W^{3}$ can mix with the new gauge bosons $W^{\prime 3}$ and $X$, all of which have even $h$ parity. Together with the Stueckelberg mass parameter $M_{X}$ for the Abelian $U(1)_{X}$, SSB generates a 4 by 4 neutral gauge boson mass matrix. In the basis of
$\left\{B, W^{3}, W^{\prime 3}, X\right\}[4,10]$, one can apply the weak rotation on upper left $2 \times 2$ block of the mass matrix, resulting in a zero eigenvalue identified as the SM photon and a 3 by 3 submatrix which can be diagonalized by an orthogonal rotation matrix $\mathcal{O}$ so that the physical states $\left(Z, Z^{\prime}, A^{\prime}\right)=$ $\left(Z^{\mathrm{SM}}, W^{\prime 3}, X\right) \cdot \mathcal{O}^{T}$. In this analysis, we arrange the neutral gauge boson masses as $m_{A^{\prime}}<m_{Z^{\prime}}<m_{Z}$ with $Z$ identified as the physical SM $Z$ boson with mass $91.1876 \pm$ $0.0021 \mathrm{GeV}$ [17]. Two more lighter neutral vector bosons, namely a dark photon $A^{\prime}$ and a dark $Z^{\prime}$, are predicted.

The new gauge couplings $g_{H}$ and $g_{X}$ for $S U(2)_{H}$ and $U(1)_{X}$ are expected to be much smaller than the SM $g$ and $g^{\prime}$ in order not to jeopardize the electroweak precision data. The VEV $v_{\Phi}$ is also expected to be larger than $v$ since all new heavy fermion masses are proportional to it. Furthermore, since we want the hierarchy $m_{A^{\prime}}<m_{Z^{\prime}}<m_{Z}$, we require $M_{X}<v$. The neutral gauge boson masses can then be well approximated by

$$
\begin{aligned}
& m_{Z}^{2} \approx m_{Z^{\text {SM }}}^{2} \equiv \frac{1}{4}\left(g^{2}+g^{\prime 2}\right) v^{2}, \\
& m_{Z^{\prime}}^{2} \approx m_{W^{\prime}}^{2}\left(1+\frac{4 g_{X}^{2}}{g_{H}^{2}}\right)+M_{X}^{2}-m_{A^{\prime}}^{2} \quad \text { and } \\
& m_{A^{\prime}}^{2} \approx M_{X}^{2}\left(1+\frac{4 g_{X}^{2}}{g_{H}^{2}}+\frac{M_{X}^{2}}{m_{W^{\prime}}^{2}}\right)^{-1} .
\end{aligned}
$$

Thus, the masses of the DM $W^{\prime(p, m)}$, dark photon $A^{\prime}$, and dark $Z^{\prime}$ are entangled with each other, and we have $m_{Z^{\prime}} \gtrsim m_{W^{\prime}}$ and $m_{A^{\prime}} \lesssim M_{X}$. Since the couplings of $A^{\prime}$ and $Z^{\prime}$ with the SM fermions are proportional to the smaller couplings $g_{H}$ and/or $g_{X}$, the Drell-Yan type processes are suppressed, and this can explain the null results of extra neutral gauge bosons searches at LEP.

It is useful to express the fundamental parameters in the scalar potential in terms of the physical scalar boson masses. Indeed, one can trade six model parameters with five physical squared masses and one mixing angle, $\left\{\lambda_{H}, \lambda_{\Phi}, \lambda_{H \Phi}, \lambda_{H \Phi}^{\prime}, \lambda_{H}^{\prime}, v_{\Phi}\right\} \rightarrow\left\{m_{h_{1}}^{2}, m_{h_{2}}^{2}, m_{D}^{2}, m_{H^{ \pm}}^{2}, m_{W^{\prime}}^{2}, \theta_{1}\right\}$. The remaining free parameters of the model are $g_{H}, g_{X}, M_{X}$, and $m_{f^{\mathrm{H}}}$.

\section{THEORETICAL CONSTRAINTS}

(a) Vacuum stability: To make sure the scalar potential is bounded from below, we follow [9] and use copositivity conditions to obtain the following constraints: $\tilde{\lambda}_{H}(\eta) \geq$ $0, \lambda_{\Phi} \geq 0$ and $\tilde{\lambda}_{H \Phi}(\xi)+2 \sqrt{\tilde{\lambda}_{H}(\eta) \lambda_{\Phi}} \geq 0$, where $\tilde{\lambda}_{H}(\eta) \equiv$ $\lambda_{H}+\eta \lambda_{H}^{\prime} \quad$ and $\quad \tilde{\lambda}_{H \Phi}(\xi) \equiv \lambda_{H \Phi}+\xi \lambda_{H \Phi}^{\prime}$ with $0 \leq \xi \leq 1$ and $-1 \leq \eta \leq 0$.

(b) Partial wave unitarity: We compute all the spinless $2 \rightarrow 2$ scattering amplitudes induced by the quartic couplings in the scalar potential and require their magnitudes to be less than $8 \pi$ [9], namely 


$$
\begin{gathered}
\left|\lambda_{H}\right|,\left|\lambda_{\Phi}\right| \leq 4 \pi,\left|\lambda_{H \Phi}\right| \leq 8 \pi,\left|\lambda_{H \Phi}^{\prime}\right|,\left|\lambda_{H}^{\prime}\right| \leq 8 \sqrt{2} \pi \\
\left|2 \lambda_{H} \pm \lambda_{H}^{\prime}\right| \leq 8 \pi,\left|\lambda_{H \Phi}+\lambda_{H \Phi}^{\prime}\right| \leq 8 \pi \\
\left|\left(\lambda_{H}+\lambda_{H}^{\prime} / 2+\lambda_{\Phi}\right) \pm \sqrt{2 \lambda_{H \Phi}^{\prime 2}+\left(\lambda_{H}+\lambda_{H}^{\prime} / 2-\lambda_{\Phi}\right)^{2}}\right| \leq 8 \pi \\
\mid\left(5 \lambda_{H}-\lambda_{H}^{\prime} / 2+3 \lambda_{\Phi}\right) \\
\pm \sqrt{\left(5 \lambda_{H}-\lambda_{H}^{\prime} / 2-3 \lambda_{\Phi}\right)^{2}+2\left(2 \lambda_{H \Phi}+\lambda_{H \Phi}^{\prime}\right)^{2}} \mid \leq 8 \pi .
\end{gathered}
$$

(c) Electroweak constraints: Following [10], we implement all the relevant constraints from electroweak precision data on the gauge sector.

\section{EXPERIMENTAL CONSTRAINTS}

\section{A. Dark photon}

The light boson $A^{\prime}$ can be treated as a dark photon and constraints from $A^{\prime} \rightarrow \bar{\ell} \ell(\ell=e, \mu)$ should be applied. Dark photon experiments constrain the size of the coupling in this decay via a parameter $\varepsilon_{\ell}$ that appears as [18]

$$
\Gamma\left(A^{\prime} \rightarrow \bar{\ell} \ell\right)=\frac{\alpha}{3} \varepsilon_{\ell}^{2} m_{A^{\prime}} \sqrt{1-\mu_{\ell}^{2}}\left(1+\frac{\mu_{\ell}^{2}}{2}\right),
$$

where $\mu_{\ell}=2 m_{\ell} / m_{A^{\prime}}<1$. In G2HDM, the parameter $\varepsilon_{\ell}$ at tree level is given by

$$
\varepsilon_{\ell}=\frac{1}{2 s_{W} c_{W}} \sqrt{\left(v_{\ell}^{A^{\prime}}\right)^{2}+\left(a_{\ell}^{A^{\prime}}\right)^{2}\left(\frac{1-\mu_{\ell}^{2}}{1+\mu_{\ell}^{2} / 2}\right)},
$$

where $v_{\ell}^{A^{\prime}}$ and $a_{\ell}^{A^{\prime}}$ are the vector and axial couplings [10]. Since $Z^{\prime}$ is also expected to be light, the dark photon experimental limits can also be applied as above with $A^{\prime} \rightarrow Z^{\prime}$ in Eq. (8) and $\left\{v_{\ell}^{A^{\prime}}, a_{\ell}^{A^{\prime}}\right\} \rightarrow\left\{v_{\ell}^{Z^{\prime}}, a_{\ell}^{Z^{\prime}}\right\}$ in Eq. (9). However, since $A^{\prime}$ is lighter, it is expected to be more strongly constrained. Typically, one finds $a_{\ell}^{A^{\prime}} \sim 10^{-3} v_{\ell}^{A^{\prime}}$ and $a_{\ell}^{Z^{\prime}} \sim 10^{-2} v_{\ell}^{Z^{\prime}}$ in our numerical scan. Since both $v_{\ell}^{A^{\prime}\left(Z^{\prime}\right)}$ and $a_{\ell}^{A^{\prime}\left(Z^{\prime}\right)}$ have the same values for all charged leptons, $\varepsilon_{\ell}$ is only weakly dependent on $\ell$ through $\mu_{\ell}$ in both cases.

Many experiments had reported stringent limits on $\varepsilon_{\ell}$ for low mass $m_{A^{\prime}}$ [19-24]. Current limits on $\varepsilon_{\ell}$ for $m_{A^{\prime}}>$ $1 \mathrm{MeV}$ are displayed on the top panel of Fig. 10 in [18].

\section{B. Higgs constraints}

In this analysis, we take the mass of the observed Higgs boson as $m_{h_{1}}=125.10 \pm 0.14 \mathrm{GeV}$ [17].
We consider two signal strengths of $h_{1} \rightarrow \gamma \gamma$ and $h_{1} \rightarrow f \bar{f}$ from gluon fusion. Their current experimental values are $\mu_{\mathrm{ggH}}^{\gamma \gamma}=0.96 \pm 0.14$ [25] and $\mu_{\mathrm{ggH}}^{\tau \tau}=1.05_{-0.47}^{+0.53}$ [26]. Besides the contributions from the SM charged particles in the 1-loop process $h_{1} \rightarrow \gamma \gamma$, we also include all charged $f^{\mathrm{H}}$ (with $m_{f^{\mathrm{H}}}$ fixed at $3 \mathrm{TeV}$ ) and $H^{ \pm}$in G2HDM.

If $m_{h_{1}}>2 m_{W^{\prime}}, h_{1}$ can decay invisibly into a pair of $W^{\prime(p, m)}$ with an invisible branching ratio $\mathrm{BR}\left(h_{1} \rightarrow\right.$ inv $)=$ $\Gamma\left(h_{1} \rightarrow W^{\prime p} W^{\prime m}\right) / \Gamma_{h_{1}}$. Recently, assuming that the Higgs boson production cross section via vector boson fusion is comparable to the SM prediction, ATLAS sets the limit $\mathrm{BR}\left(h_{1} \rightarrow\right.$ inv $)<0.13$ at $95 \%$ C.L. [27].

\section{Dark matter constraints}

\section{Relic density}

The main DM annihilation channels in our model are to pairs of SM fermions mediated by $Z$ and $Z^{\prime}$. Other annihilation channels are also possible but are far more suppressed. First, there is also the $A^{\prime}$ exchange diagram. The $A^{\prime}$ couplings to SM fermions are suppressed by combinations of new gauge couplings in $v_{f}^{A^{\prime}}$ and $a_{f}^{A^{\prime}}$. Similarly to the case of $A^{\prime}$, the $Z^{\prime}$ couplings to the SM fermions are suppressed by its own $v_{f}^{Z^{\prime}}$ and $a_{f}^{Z^{\prime}}$. However, it is possible to have $\sqrt{s} \approx 2 m_{W^{\prime}} \approx m_{Z^{\prime}}$, resulting in an important contribution from resonant annihilation. Secondly, we also have the $h_{1}$ and $h_{2}$ Higgs exchange diagrams. Their couplings to pairs of $W^{\prime(p, m)}$ and SM fermions are suppressed by $g_{H}^{2}$ and light fermion masses $m_{q} / v$, respectively. Finally, a $t$-channel diagram via an exchange of $f^{\mathrm{H}}$ is suppressed by $g_{H}^{2}$ and the mass of the new heavy fermion in the propagator. The annihilation mediated by the $Z$ is also suppressed by a mixing angle of $\mathcal{O}_{21}^{2}$ that is required to be small, mostly by measurements on the total decay width of the $Z$ and branching fraction for the invisible decay $Z \rightarrow W^{\prime p} W^{\prime m}$. As mentioned above, the channel mediated by $Z^{\prime}$ is also suppressed by $g_{H}$ and $g_{X}$ inside the couplings of $v_{f}^{Z^{\prime}}$ and $a_{f}^{Z^{\prime}}$. However, these suppressions are not as severe as in other channels, and when we include the effects from $Z^{\prime}$ resonance, it is possible to bring the relic density to its observed value of $\Omega_{\mathrm{DM}} h^{2}=0.120 \pm$ 0.001 from Planck's measurement [28].

\section{Direct detection}

Due to the small couplings between the DM candidate, $W^{\prime(p, m)}$, with the SM-like states $h_{1}$ and $Z$ and the new states $h_{2}, Z^{\prime}$, and $A^{\prime}$, which couple to the visible sector, it is possible to have effects from DM scattering against nucleons in detectors used in direct detection experiments. In this case, we have to consider the elastic scattering between a DM particle and the quarks inside the nucleon. The suppressions from $h_{1,2}\left(\right.$ and $\left.f^{\mathrm{H}}\right)$ exchange work in the 
same way as in the processes for relic density just described. Therefore, we are only left with the processes mediated by $Z, Z^{\prime}$, and $A^{\prime}$ in the $t$ channel. Usually, for direct detection processes, the momentum exchange $|\mathbf{q}|$ is rather small. This will result in amplitudes suppressed by the inverse mass squared of the mediator meaning that the lighter states, $Z^{\prime}$ and $A^{\prime}$, will be less suppressed than the $Z$. In the approximation that $|\mathbf{q}| \ll m_{Z(i)}$, the interaction between $W^{\prime}$ and a light quark $q$ can be written as a contact interaction,

$$
\begin{aligned}
\mathcal{L}_{\mathrm{CI}-\mathrm{DD}}= & \sum_{q} \sum_{i=2}^{3} \frac{g_{M} g_{H} \mathcal{O}_{2 i} v_{q}^{Z(i)}}{2 m_{Z(i)}^{2}} \\
& \times\left(W^{\prime p \mu} \partial_{\nu} W_{\mu}^{\prime m}-W^{\prime m \mu} \partial_{\nu} W_{\mu}^{\prime p}\right) \bar{q} \gamma^{\nu} q,
\end{aligned}
$$

where $Z(2) \equiv Z^{\prime}$ and $Z(3) \equiv A^{\prime}$. It is worth noting that, as light as the mediators $Z^{\prime}$ and $A^{\prime}$ are, we can still integrate them out thanks to the comparably small maximum momentum transfer, $|\mathbf{q}|_{\max }$. The smallness of $|\mathbf{q}|_{\max }$ is also due to $W^{\prime}$ being light. Consider $|\mathbf{q}|_{\text {max }}=$ $2 v_{\mathrm{DM}} m_{W^{\prime}} m_{A} /\left(m_{W^{\prime}}+m_{A}\right) \quad$ with $\quad v_{\mathrm{DM}}=10^{-3} c, \quad m_{W^{\prime}}=$ $0.5 \mathrm{GeV}$ and the target mass $m_{A}=131 \mathrm{GeV}$ or $40 \mathrm{GeV}$ for xenon or argon target, respectively. In both cases, $|\mathbf{q}|_{\max } \sim O(1 \mathrm{MeV})$, while we expect $m_{A^{\prime}} \gtrsim O(10 \mathrm{MeV})$ due to constraints on dark photon. Smaller $m_{W^{\prime}}$ results in even smaller $|\mathbf{q}|_{\max }$. Furthermore, for the smaller axial couplings with the quarks, in the small momentum exchange limit, only the space components of $\gamma^{\nu}$ remain, which are suppressed by the $W^{\prime(p, m)}$ momentum due to the derivatives $\nabla W^{\prime(p, m)}$ as in (10) $[29,30]$.

From Eq. (10), it is clear that the $A^{\prime}$ mediated process is expected to dominate the cross section unless $\left|\mathcal{O}_{23} / \mathcal{O}_{22}\right|<\left|m_{A^{\prime}} / m_{Z^{\prime}}\right|^{2}$. The case where both mediators participate equally is expected to happen only through fine tuning of masses and mixings. Therefore, we expect the cross section with the nucleons to be mostly mediated by either $A^{\prime}$ or $Z^{\prime}$. The elastic spin-independent (SI) cross section between $W^{\prime(p, m)}$ and a nucleon, $N$, is given by [31]

$$
\begin{gathered}
\sigma_{W^{\prime} N}^{\mathrm{SI}}=\sigma_{W^{\prime} p}^{\mathrm{SI}} \frac{\sum_{k} \eta_{k} \mu_{A_{k}}^{2}\left[Z_{\text {atom }}+\left(A_{k}-Z_{\text {atom }}\right) f_{n} / f_{p}\right]^{2}}{\sum_{k} \eta_{k} \mu_{A_{k}}^{2} A_{k}^{2}}, \\
\sigma_{W^{\prime} p}^{\mathrm{SI}}=\frac{\mu_{p}^{2} g_{M}^{2} g_{H}^{2} \mathcal{O}_{2 i}^{2}}{4 \pi m_{Z(i)}^{4}} f_{p}^{2}, \quad(i=2 \text { or } 3) .
\end{gathered}
$$

where $\mu_{p}=m_{W^{\prime}} m_{p} /\left(m_{W^{\prime}}+m_{p}\right)$ is the reduced DMproton mass, $\mu_{A_{k}}=m_{W^{\prime}} m_{A_{k}} /\left(m_{W^{\prime}}+m_{A_{k}}\right)$ is the reduced DM-isotope nucleus mass, and $f_{p}$ and $f_{n}$ are effective couplings of the DM with protons and neutrons, respectively. $Z_{\text {atom }}$ is the atomic number, and the isotope dependent variables $\eta_{k}$ and $A_{k}$ are the abundance and mass number of the $k^{\text {th }}$ target isotope, respectively. Direct detection experiments usually report the number in
Eq. (11), assuming isospin conservation, i.e., $f_{p}=f_{n}$. In that case, it is straightforward to see that the ratio of the sums over isotopes reduces to 1 and $\sigma_{W^{\prime} N}^{S I}=\sigma_{W^{\prime} p}^{S I}$. However, in our case, the couplings between quarks, $u$ and $d$, and the gauge bosons, $Z^{\prime}$ and $A^{\prime}$, are all different due to their distinct SM charges leading to isospin violation (ISV), i.e., $f_{p} \neq f_{n}$. Following [31,32], we can rescale the reported experimental limit, $\sigma_{\text {limit }} \rightarrow \sigma_{\text {limit }} \times \sigma_{W^{\prime} p}^{\text {SI }} / \sigma_{W^{\prime} N}^{\text {SI }}$ to account for ISV effects and use it to limit $\sigma_{W^{\prime} p}^{\mathrm{SI}}$ as given by Eq. (12). This rescaling depends on the mass of DM, the atomic numbers and the ratio $f_{n} / f_{p}$, and, hence, will be different for different points in the parameter space. To constraint $\sigma_{W^{\prime} p}^{\mathrm{SI}}$, we use the most recent limits set by CRESST III [33], DarkSide-50 [34], and XENON1T [35].

\section{Indirect detection}

Due to DM annihilation in SM particles before freezeout happening through the resonance of an otherwise suppressed channel, the annihilation of DM at the present time-after the shift in energy from the early Universe to the current time-loses the resonance resulting in a very low annihilation cross section. We have checked that the value of the total annihilation cross section in G2HDM at the present time is of order $10^{-32} \mathrm{~cm}^{3} \cdot \mathrm{s}^{-1}$ or below, much lower than the canonical limits set for various channels by Fermi-LAT data [36,37].

\section{Monojet}

The event of an energetic jet with large missing transverse momentum has been searched by ATLAS $[38,39]$ and CMS [40] Collaborations with null results. In G2HDM, the process $p p \rightarrow W^{\prime p} W^{\prime m} j$ can give rise to the monojet signal at the LHCIn the most sensitive signal region with $E_{T}^{\text {miss }} \in$ $(700,800) \mathrm{GeV}$ (the signal region EM7 in [39]), we have checked that at a couple of benchmark points (BP1 and 2), the monojet signals with $p_{T}^{\text {miss }}>100 \mathrm{GeV}$ and precuts for jets $p_{T}^{j}>30 \mathrm{GeV}$ and $\left|\eta_{j}\right|<2.8$, are at least 2 orders of magnitude below the current 95\% C.L. exclusion limit on the production cross section of $400 \mathrm{fb}$ and $680 \mathrm{fb}$, respectively [39], as shown in Table I.

\section{METHODOLOGY}

To sample the parameter space, we use the affine invariant Markov chain Monte Carlo (MCMC) ensemble sampler emcee [41], which presents advantages such as fast calculation of parameter distributions in multidimensions. The initial priors and ranges of the free parameters used in our scan are tabulated in Table II.

The DM constraints from the relic density, direct detection, and indirect detection are calculated using micrOMEGAs [42] and a set of model files generated by FeynRules [43]. For the invisible decay branching ratio of the 
TABLE I. Parameters for the two benchmark points where the production cross section of the monojet signals with precuts are computed, as shown in the last column.

\begin{tabular}{lccccccccc}
\hline \hline BP & $m_{W^{\prime}}(\mathrm{GeV})$ & $M_{X}(\mathrm{GeV})$ & $m_{h_{2}}(\mathrm{TeV})$ & $m_{D}(\mathrm{TeV})$ & $m_{H^{ \pm}}(\mathrm{TeV})$ & $g_{H}\left(10^{-4}\right)$ & $g_{X}\left(10^{-4}\right)$ & $\theta_{1}(\mathrm{rad})$ & $\sigma_{p p \rightarrow W^{\prime p} W^{\prime m} j}^{\text {precut }}(\mathrm{fb})$ \\
\hline 1 & 1.0 & 1.67 & 0.79 & 2.0 & 2.12 & 5.4 & 3.2 & 0.17 & 3.0 \\
2 & 0.17 & 0.33 & 2.9 & 1.54 & 1.64 & 1.0 & 0.4 & 0.11 & 3.8 \\
\hline \hline
\end{tabular}

Higgs boson, we take advantage of the use of CalcHEP [44] within micrOMEGAs to calculate the decay width along with the rest of the DM constraints mentioned previously.

All the points outside the theoretical constraints are simply rejected. By the same token, the dark photon constraints are used to reject any parameter combination of $\varepsilon_{e, \mu}-m_{A^{\prime}}$ or $\varepsilon_{e, \mu}-m_{Z^{\prime}}$ located inside the currently excluded regions. As mentioned earlier, if the G2HDM predictions for the DM indirect detection rates and monojet events are at least 1-2 orders of magnitude below the current experimental limits, we will not include them in our present analysis. The rest of the constraints are summed into a total $\chi^{2}$ that also includes relic density and direct detection spin-independent (SI) cross section. In the case of direct detection experiments, in mass ranges where more than one limit exists, we consider only the strongest constraint. Note that, due to isospin violation (ISV), the most constraining result for direct detection may not correspond to the experiment with the smallest cross section. Since direct detection limits are reported assuming $f_{p}=f_{n}$ in Eq. (11), it is possible for ISV $\left(f_{p} \neq f_{n}\right)$ to produce some amount of cancellation or enhancement of the limits depending on the atoms used in the detector. Computing the SI cross section in the manner described in Sec. IV C allows us to account for ISV and the different atoms used in different experiments. For direct detection and Higgs invisible decay limits, reported at $95 \%$ C.L., we add the $\chi^{2}$ contributions $\chi_{\text {DD }}^{2}=2.71 \times\left(\sigma_{\text {theory }} / \sigma_{\text {limit }}\right)^{2}$ and $\chi_{\text {inv }}^{2}=2.71 \times\left(\operatorname{BR}\left(h_{1} \rightarrow\right.\right.$ inv) $/ 0.13)^{2}$, respectively. The 2.71 factor allows for $\chi^{2}=$ 2.71 when our prediction is exactly at the reported 95\% C.L. limits with null-signal assumption.

\section{RESULTS}

Figure 1 shows the allowed region projected on the $\left(m_{W^{\prime}}, g_{H}\right)$ plane, where the band-shaped region is caused

TABLE II. Ranges and values for the prior of eight free parameters. We fix $m_{f^{\mathrm{H}}}=3 \mathrm{TeV}$.

\begin{tabular}{lc}
\hline \hline Parameter [unit] & Range \\
\hline$m_{h_{2}}, m_{D}, m_{H^{ \pm}}[\mathrm{TeV}]$ & {$[0.3,10]$} \\
$\log _{10}\left(m_{W^{\prime}} / \mathrm{GeV}\right), \log _{10}\left(M_{X} / \mathrm{GeV}\right)$ & {$[-3,2]$} \\
$\log _{10}\left(g_{H}\right), \log _{10}\left(g_{X}\right)$ & {$[-6,0]$} \\
$\theta_{1}[\mathrm{rad}]$ & {$[-\pi / 2, \pi / 2]$} \\
\hline \hline
\end{tabular}

by the relation $\Omega_{\mathrm{DM}} h^{2} \propto 1 /\langle\sigma v\rangle$. Since $\sigma \propto g_{H}^{2} m_{W^{\prime}}^{2} / s^{2}$, for $s \sim 4 m_{W^{\prime}}^{2}$ we have $g_{H}^{2} m_{W^{\prime}}^{2} / s^{2} \sim g_{H}^{2} / 16 m_{W^{\prime}}^{2}$ resulting in $\Omega_{\mathrm{DM}} h^{2} \propto m_{W^{\prime}}^{2} / g_{H}^{2}$. In order to have a constant relic density, $m_{W^{\prime}}$ and $g_{H}$ have to maintain a linear relation as displayed in the plot. Deviation from this band results in a relic density lying outside Planck's allowed range.

Note that the allowed region in Fig. 1 are bounded in their top-right and bottom-left corners by direct detection (DD) and the constraint on dark photon, respectively. We know that the direct detection cross section grows with $g_{H}^{2}$ as seen in Eq. (12); therefore, it is expected to see it setting an upper bound on $g_{H}$. In the bottom-left region disfavored by dark photon searches, this is mostly due to the constraint from $\nu$-CAL I, which limits $\varepsilon$ from below as will be explained in Fig. 2.

In the left panel of Fig. 2, we show the allowed region projected on the $\left(m_{W^{\prime}}, \sigma_{W^{\prime} p}^{S I}\right)$ plane. The dark (light) blue shaded zone represents the $1 \sigma(2 \sigma)$ allowed region. The current DM direct detection measurements from CRESST III (green) [33], DarkSide-50 (orange) [34], and XENON1T (brown) [35] constrain the DM mass to remain below $\sim 2 \mathrm{GeV}$. A small part of the $2 \sigma$ allowed region lies below the neutrino floor (light orange), where the coherent neutrino-nucleus scattering would

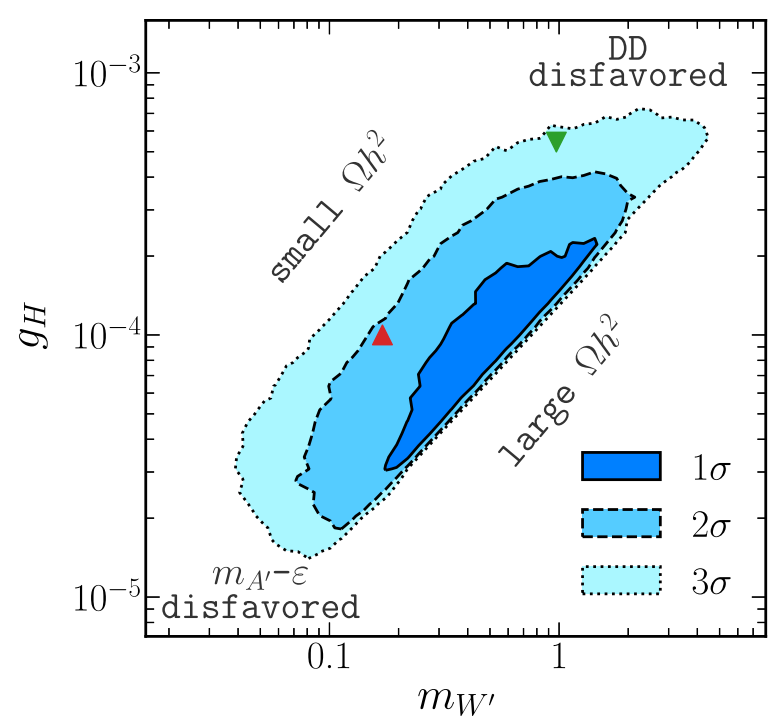

FIG. 1. The $1 \sigma, 2 \sigma$, and $3 \sigma$ allowed regions projected on the $\left(m_{W^{\prime}}, g_{H}\right)$ plane. Benchmark points used for monojet analysis are also projected as indicated by the green down triangle (BP1) and red up triangle $(\mathrm{BP} 2)$. 

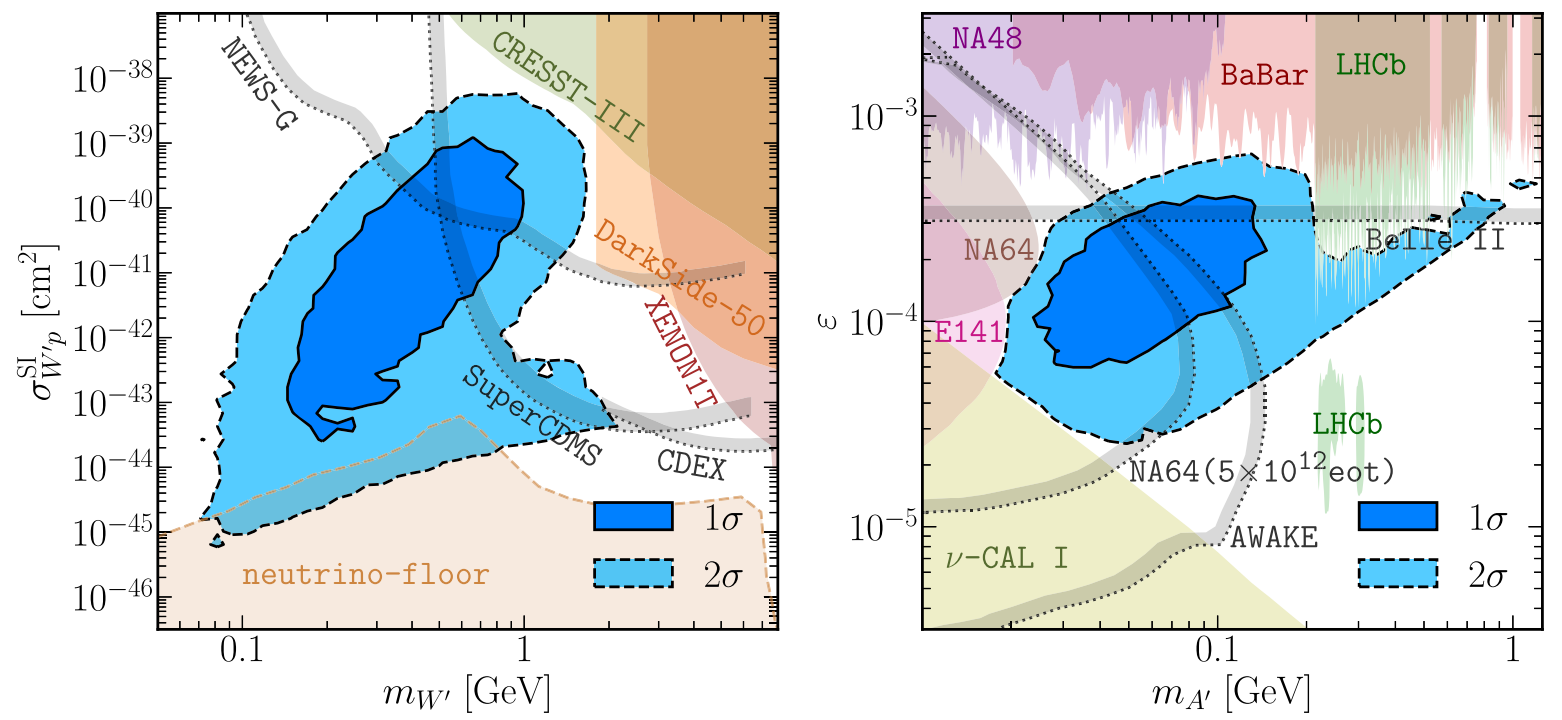

FIG. 2. The $1 \sigma$ and $2 \sigma$ allowed contours projected on the $\left(m_{W^{\prime}}, \sigma_{W^{\prime} p}^{\text {SI }}\right)$ plane (left) and the $\left(m_{A^{\prime}}, \varepsilon\right)$ plane (right). The experimental excluded regions used are shown as solid colored regions. Projected experimental limits are shown as dotted lines.

dominate over any DM signal. Additionally, we show that experiments in the near future such as NEWS-G [45], SuperCDMS [46], and CDEX [47] can further probe our allowed parameter space, in particular, $m_{W^{\prime}}$ can reach $\gtrsim 0.3 \mathrm{GeV}$ with NEWS-G and $\sigma_{W^{\prime} p}^{\text {SI }}$ can push down to about $10^{-44} \mathrm{~cm}^{2}$ with SuperCDMS and CDEX.

The right panel in Fig. 2 shows the allowed region projected on the $\left(m_{A^{\prime}}, \varepsilon\right)$ plane. Various experimental limits from dark photon searches are displayed in color shaded zones including LHCb (green) [19], BABAR (pink) [20], NA48 (purple) [21], NA64 (light brown) [22], E141 (magenta) [23], and $\nu$-CAL I (olive green) [24]. The dilepton searches at the LHCb, BABAR, and NA48 put upper limits of $\varepsilon \lesssim 10^{-3}$ for $m_{A^{\prime}} \gtrsim 0.03 \mathrm{GeV}$, especially $\mathrm{LHCb}$, which sets a strong limit on $\varepsilon$ at $0.2 \mathrm{GeV}<m_{A^{\prime}}<$ $0.5 \mathrm{GeV}$ causing a concave region in the $2 \sigma$ allowed region at this mass range. We note that this concave region due to LHCb corresponds to the right-tilted concave region at $\left(m_{W}^{\prime}, \sigma_{W^{\prime}}^{\mathrm{SI}}\right) \sim\left(1 \mathrm{GeV}, 10^{-42} \mathrm{~cm}^{2}\right)$ in the left panel of the same figure. Experimental probes of dark photon, dark $Z^{\prime}$, and dark matter are thus correlated. The LHCb long-lived dark photon search constraints [19] are also shown by the two isolated green shaded islands.

On the other hand, the beam dump experiments NA64, E141, and $\nu$-CAL I close the available space for smaller $\varepsilon$ and lighter $m_{A^{\prime}}$ setting lower bounds of $m_{A^{\prime}}>0.02 \mathrm{GeV}$ and $\varepsilon \gtrsim 2 \times 10^{-5}$. The lower limit on $\varepsilon$ for $m_{A^{\prime}}>0.05 \mathrm{GeV}$ is due to the DM relic density measured by the Planck's experiment.

Interestingly, our final allowed region is located in the gap between the beam-dump and the collider based experiments, an area of special interest for future dark photon searches. For instance, Belle-II [48] with a luminosity of
$50 \mathrm{ab}^{-1}$ can probe $\varepsilon$ down to $2 \times 10^{-4}$, the next upgrade of NA64 [49] can cover $10^{-5} \lesssim \varepsilon \lesssim 10^{-3}$ and $m_{A^{\prime}} \lesssim 0.08 \mathrm{GeV}$ by reaching $\sim 5 \times 10^{12}$ electrons-on-target (abbreviated by eot in the figure), and Advanced WAKEfield Experiment (AWAKE) run 2 [50] can reach $m_{A^{\prime}}$ up to $0.15 \mathrm{GeV}$ with $10^{16}$ electrons-on-target with an energy of $50 \mathrm{GeV}$. These limits are shown explicitly in the right panel of Fig. 2. In the future, with access to high energy electron-proton colliders, AWAKE may reach $1 \mathrm{TeV}$ for the electrons, extending $m_{A^{\prime}}$ up to $0.6 \mathrm{GeV}$ [50].

\section{CONCLUSIONS}

G2HDM is based on a simple but novel idea of gauging the inert Higgs doublet into an appealing SM-like gauge group of $S U(2)_{H} \times U(1)_{X}$ in the hidden sector. We summarize our findings as follows:

(i) A remnant $h$ parity emerges to stabilize $W^{\prime(p, m)}$ as a DM candidate, which, unlike the left-right model, does not mix with the $\mathrm{SM} W^{ \pm}$and hence, relieves us from the severe experimental constraint of the leftright symmetry breaking scale. Also, the $h$ parity ensures naturally the absence of tree level flavor changing neutral current in the SM sector.

(ii) A low mass gauge sector of $W^{\prime}$, dark photon, and $Z^{\prime}$ in the sub-GeV range with a superweak size couplings $g_{H}$ and $g_{X}$ of $O\left(10^{-5}-10^{-3}\right)$ are consistent with relic density and all experimental constraints. While the dark $Z^{\prime}$ can be the dominant resonant contribution for DM relic density, the dark photon is crucial for DM direct detection. Besides the possibility of detecting a low mass $W^{\prime}$ in direct detection experiments, the dark photon is predicted to be well positioned for future probes. 
(iii) Due to the superweak couplings, direct searches for the dark gauge sector as well as other new particles in G2HDM at the LHC or future colliders are in both the long lifetime and high energy/luminosity frontiers, which deserve further studies.

Experimental searches for low mass non-Abelian $W^{\prime} \mathrm{DM}$, dark photon, and dark $Z^{\prime}$ in the sub-GeV range are complementary with each other, and the results we presented here should be of general interests to both experimentalists and model builders.

\section{ACKNOWLEDGMENTS}

The analysis presented here was done using the resources of the high-performance T3 Cluster at the Institute of Physics, Academia Sinica. This work is supported in part by the Ministry of Science and Technology of Taiwan under Grants No. 108-2112-M-001-018 (TCY) and No. 108-2811-M-001-550 (RR) and by National Natural Science Foundation of China under Grants No. 11775109 and No. U1738134 (VQT).
[1] A. H. Chamseddine, R. L. Arnowitt, and P. Nath, Phys. Rev. Lett. 49, 970 (1982); Phys. Lett. B 121, 33 (1983).

[2] H. P. Nilles, Phys. Rep. 110, 1 (1984).

[3] G. Jungman, M. Kamionkowski, and K. Griest, Phys. Rep. 267, 195 (1996).

[4] W. C. Huang, Y. L. S. Tsai, and T. C. Yuan, J. High Energy Phys. 04 (2016) 019.

[5] C. R. Chen, Y. X. Lin, C. S. Nugroho, R. Ramos, Y. L. S. Tsai, and T. C. Yuan, Phys. Rev. D 101, 035037 (2020).

[6] R. N. Mohapatra and G. Senjanovic, Phys. Rev. Lett. 44, 912 (1980); Phys. Rev. D 23, 165 (1981).

[7] S. L. Glashow and S. Weinberg, Phys. Rev. D 15, 1958 (1977).

[8] G. C. Branco, P. M. Ferreira, L. Lavoura, M. N. Rebelo, M. Sher, and J. P. Silva, Phys. Rep. 516, 1 (2012).

[9] A. Arhrib, W. C. Huang, R. Ramos, Y. L. S. Tsai, and T. C. Yuan, Phys. Rev. D 98, 095006 (2018).

[10] C. T. Huang, R. Ramos, V. Q. Tran, Y. L. S. Tsai, and T. C. Yuan, J. High Energy Phys. 09 (2019) 048.

[11] C. D. Carone and R. Ramos, Phys. Rev. D 88, 055020 (2013).

[12] H. Davoudiasl and I. M. Lewis, Phys. Rev. D 89, 055026 (2014).

[13] S. Baek, P. Ko, and W. I. Park, J. Cosmol. Astropart. Phys. 10 (2014) 067.

[14] B. Barman, S. Bhattacharya, S. K. Patra, and J. Chakrabortty, J. Cosmol. Astropart. Phys. 12 (2017) 021.

[15] B. Barman, S. Bhattacharya, and M. Zakeri, J. Cosmol. Astropart. Phys. 02 (2020) 029.

[16] T. Abe, M. Fujiwara, J. Hisano, and K. Matsushita, J. High Energy Phys. 07 (2020) 136.

[17] P. A. Zyla et al. (Particle Data Group), Prog. Theor. Exp. Phys. (2020), 083C01.

[18] M. Fabbrichesi, E. Gabrielli, and G. Lanfranchi, arXiv: 2005.01515.

[19] R. Aaij et al. (LHCb Collaboration), Phys. Rev. Lett. 124, 041801 (2020).

[20] J. P. Lees et al. (BABAR Collaboration), Phys. Rev. Lett. 113, 201801 (2014).

[21] J. R. Batley et al. (NA48/2 Collaboration), Phys. Lett. B 746, 178 (2015).

[22] D. Banerjee et al. (NA64 Collaboration), Phys. Rev. Lett. 120, 231802 (2018).
[23] E. M. Riordan et al., Phys. Rev. Lett. 59, 755 (1987).

[24] J. Blümlein and J. Brunner, Phys. Lett. B 701, 155 (2011); Phys. Lett. B 731, 320 (2014).

[25] G. Aad et al. (ATLAS Collaboration), Phys. Rev. D 101, 012002 (2020).

[26] A. M. Sirunyan et al. (CMS Collaboration), Eur. Phys. J. C 79, 421 (2019).

[27] ATLAS Collaboration, Report No. ATLAS-CONF-2020-008.

[28] N. Aghanim et al. (Planck Collaboration), Astron. Astrophys. 641, A6 (2020).

[29] M. Escudero, A. Berlin, D. Hooper, and M. X. Lin, J. Cosmol. Astropart. Phys. 12 (2016) 029.

[30] G. Arcadi, M. Dutra, P. Ghosh, M. Lindner, Y. Mambrini, M. Pierre, S. Profumo, and F. S. Queiroz, Eur. Phys. J. C 78, 203 (2018).

[31] J. L. Feng, J. Kumar, D. Marfatia, and D. Sanford, Phys. Lett. B 703, 124 (2011).

[32] C. E. Yaguna, Phys. Rev. D 95, 055015 (2017).

[33] G. Angloher et al. (CRESST Collaboration), Eur. Phys. J. C 77, 637 (2017).

[34] P. Agnes et al. (DarkSide Collaboration), Phys. Rev. Lett. 121, 081307 (2018).

[35] E. Aprile et al. (XENON Collaboration), Phys. Rev. Lett. 123, 251801 (2019).

[36] M. Ackermann et al. (Fermi-LAT Collaboration), Phys. Rev. Lett. 115, 231301 (2015).

[37] A. Albert et al. (Fermi-LAT and DES Collaborations), Astrophys. J. 834, 110 (2017).

[38] M. Aaboud et al. (ATLAS Collaboration), J. High Energy Phys. 01 (2018) 126.

[39] ATLAS Collaboration, Report No. ATLAS-CONF-2020048.

[40] A. M. Sirunyan et al. (CMS Collaboration), J. High Energy Phys. 07 (2017) 014.

[41] D. Foreman-Mackey, D. W. Hogg, D. Lang, and J. Goodman, Publ. Astron. Soc. Pac. 125, 306 (2013).

[42] G. Bélanger, F. Boudjema, A. Goudelis, A. Pukhov, and B. Zaldivar, Comput. Phys. Commun. 231, 173 (2018).

[43] A. Alloul, N. D. Christensen, C. Degrande, C. Duhr, and B. Fuks, Comput. Phys. Commun. 185, 2250 (2014).

[44] A. Belyaev, N. D. Christensen, and A. Pukhov, Comput. Phys. Commun. 184, 1729 (2013).

[45] M. Battaglieri et al. arXiv:1707.04591. 
[46] R. Agnese et al. (SuperCDMS Collaboration), Phys. Rev. D 95, 082002 (2017).

[47] H. Ma et al. (CDEX Collaboration), J. Phys. Conf. Ser. 1342, 012067 (2019).

[48] E. Kou et al. (Belle-II Collaboration), Prog. Theor. Exp. Phys. (2019), 123C01; (2020), 029201(E).
[49] D. Banerjee et al., Report No. CERN-SPSC-2018-004 (SPSC-P-348-ADD-2).

[50] A. Caldwell, J. Chappell, P. Crivelli, E. Depero, J. Gall, S. Gninenko, E. Gschwendtner, A. Hartin, F. Keeble, J. Osborne et al., arXiv:1812.11164. 